\title{
Multi-trigger resist - Novel synthesis improvements for high resolution EUV lithography
}

\author{
G. O’Callaghan, ${ }^{\text {b,d }}$, C. Popescu ${ }^{\mathrm{a}, \mathrm{b}, \mathrm{d}}$, A McClelland ${ }^{\mathrm{d}}$, D Kazazis, ${ }^{\mathrm{c}}, \mathrm{J}$ Roth, ${ }^{\mathrm{e}}, \mathrm{W}$ Theis, ${ }^{\mathrm{a}}$, Y Ekincic, \\ Alex P.G. Robinson., \\ ${ }^{a}$ Nanoscale Physics Research Laboratory, School of Physics and Astronomy, The University of \\ Birmingham, Birmingham, B15 2TT, UK \\ ${ }^{\mathrm{b}}$ School of Chemical Engineering, University of Birmingham, Birmingham, B15 2TT, UK \\ ${ }^{\mathrm{d}}$ Nano-C Inc., 33 Southwest Park, Westwood, MA 02090, USA \\ c Laboratory for Micro and Nanotechnology, Paul Scherrer Institut, 5232 Villigen PSI, Switzerland \\ ${ }^{\mathrm{d}}$ Irresistible Materials Ltd, Birmingham Research Park, Birmingham, UK \\ e Nano-C Inc., 33 Southwest Park, Westwood, MA 02090, USA
}

e-mail: a.p.g.robinson@bham.ac.uk

\begin{abstract}
Irresistible Materials (IM) is developing novel resist systems based on the Multi-trigger concept, which incorporates a dose dependent quenching-like behaviour. The Multi Trigger Resist is a non-metal based negative tone resist, and consists of a base molecule and a crosslinker, which represent the resist matrix, together with a photoacid generator (PAG). Previously presented MTR2 showed $16 \mathrm{~nm}$ half pitch lines patterned with a dose of $38 \mathrm{~mJ} / \mathrm{cm}^{2}$, giving a LER of $3.7 \mathrm{~nm}$ on the NXE3300. Since then, research has been undertaken to improve this resist. In particular we are focusing on improved RLS; reduced top-loss and wiggling at high aspect ratios; eliminating the antimony PAG and further reduction of chemical stochastics. In this study, we present the approaches that have been taken to attain these goals and the initial results. Using the EUV Interference Lithography tool at PSI, a multi trigger resist with a high absorbance non-metal element included in the resist matrix, MTR2627, has been patterned at a pitch of $28 \mathrm{~nm}$ with an estimated dose of $53 \mathrm{~mJ} / \mathrm{cm}^{2}$ and LER of $4.2 \mathrm{~nm}$. The LWR is improved in the low dose region, and results also show that a thicker film can be used without pattern collapse below pitch 32nm due to increased stiffness. Using the Berkeley MET tool, this resist matrix with a higher MTR ratio has patterned $24 \mathrm{~nm}$ lines at a pitch of $48 \mathrm{~nm}$ with an LER of $1.9 \mathrm{~nm}$ with a dose of $65 \mathrm{~mJ} / \mathrm{cm}^{2}$. Additionally, we present initial results for an MTR resist series where the antimony PAG has been replaced with a carbon based PAG.
\end{abstract}

Keywords: EUV lithography, photoresist, molecular resist, multi-trigger resist, chemical amplification, crosslinking

\section{INTRODUCTION}

The forthcoming introduction of EUV lithography into high volume manufacturing represents a large challenge for many aspects of the lithography process, with a suitable EUV photoresist high on the list of requirements. Whilst traditional chemically amplified resists will likely support the initial insertion, a wide range of materials options are being examined for future nodes [1-3], aiming to identify a photoresist that simultaneously meets the resolution, line edge roughness and 
sensitivity requirements, with minimal defectivity. However, these parameters are linked by a fundamental trade-off in lithography (the RLS triangle) and it is difficult to overcome. For instance, addition of quenchers in chemically amplified resists (CAR) reduces the acid diffusion length and increases the resolution of the patterned features, but decreases the sensitivity, and impacts on material stochastics affecting the line edge roughness. Defectivity due to line collapse, bridging or line breaks is also a fundamental problem.

Irresistible Materials (IM) is developing novel resist systems based on the multi-trigger concept. In a multi-trigger resist multiple elements of the resist must be simultaneously activated to enable the catalytic reactions to proceed. In high dose areas the resist therefore behaves like a traditional CAR, whilst in low dose areas, such as line edges, the reaction is secondorder increasing the chemical gradient. Effectively there is a dose dependent quenching-like behaviour built in to the resist, enhancing chemical contrast and thus resolution and reducing roughness, whilst eliminating the materials stochastics impact of a separate quencher.

In a multi-trigger material, resist exposure proceeds via a catalytic process in a similar manner to a chemically amplified resist. However, instead of a single photoacid causing a single resist exposure event and then being regenerated, in the multi-trigger resist multiple photoacids activate multiple acid sensitive molecules, which then react with each other to cause a single resist event while also regenerating the photoacids. In areas with a high number of activated photoacids (higher dose areas, for instance at the centre of a pattern feature) resist components are activated in close proximity and the multistep resist exposure reaction proceeds, ending with photoacids regeneration and thus further reactions, ensuring high sensitivity. In areas with a low number of activated photoacids (lower dose areas, for instance at the edge of a pattern feature), the activated resist components are too widely separated to react and the photoacids are not released and are thus removed, stopping the catalytic chain. The multi-trigger resist creates an increase in the chemical gradient at the edge of patterned features, and reduces undesirable acid diffusion out of the patterned area. The picture in figure 1 details this concept.
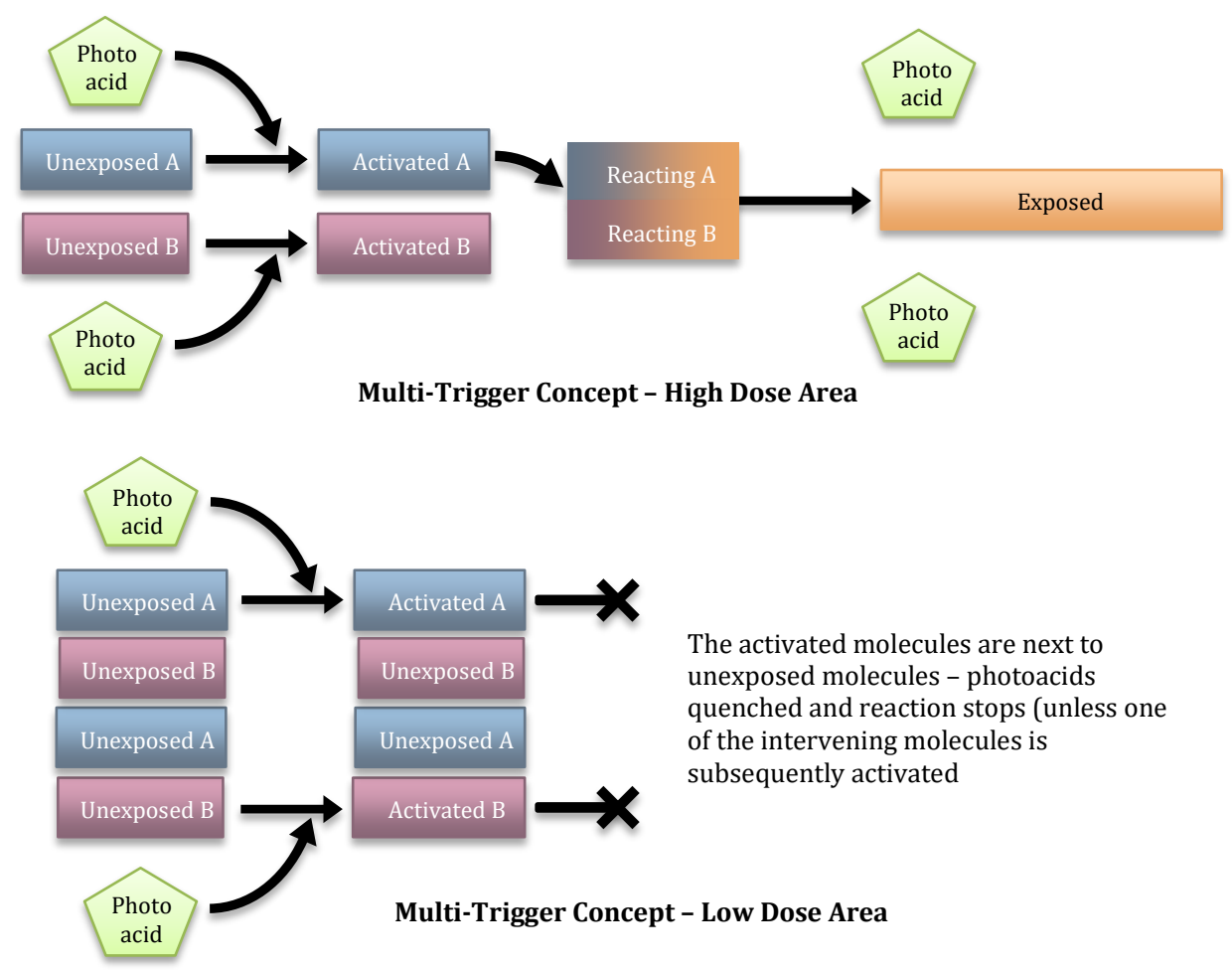

Figure 1:Multi Trigger Concept

The multi-trigger material previously presented [4-6] consists of a base molecule and a crosslinker, which represent the resist matrix, together with a photoacid generator (PAG). MTR2 showed $16 \mathrm{~nm}$ half pitch lines patterned with a dose of 
$38 \mathrm{~mJ} / \mathrm{cm}^{2}$, giving a LER of $3.7 \mathrm{~nm}$ on the NXE3300 [4]. Since then, research has been undertaken to improve this resist. In particular we are focusing on improved RLS; reduced wiggling at high aspect ratios; eliminating the antimony PAG and further reduction of chemical stochastics.

One aspect we want to address is to increase the crosslinking, however the initial MTR variant molecules have opposing behavior on Post Exposure Bake, which is the primary current limitation, as shown in figure 2, because the LER increases. By introducing higher energy leaving groups onto molecule A, we believe that we can enhance crosslinking without increasing the LER. The first example of this is presented in this study as MTR4.

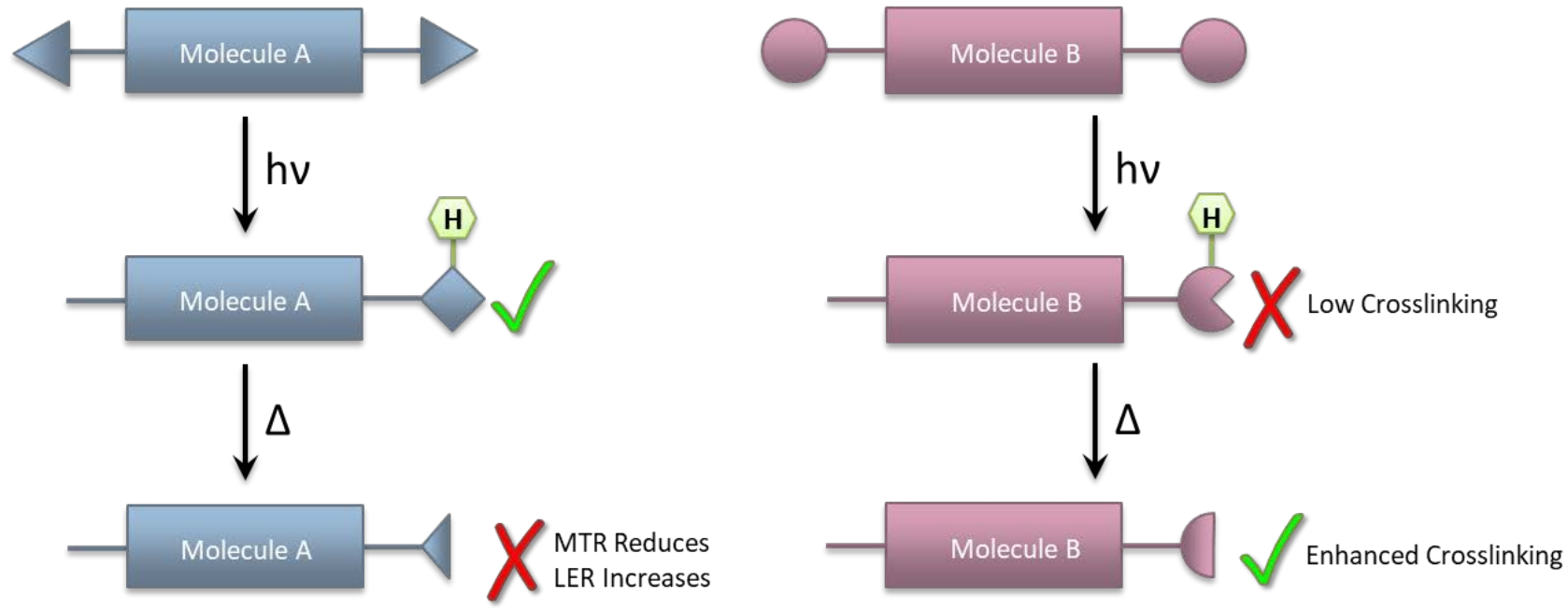

Figure 2: Showing the influence of a post exposure bake on the MTR resist matrix

Higher absorption is a potential route to overcome the photon shot noise limit in EUV lithography as well as potentially improving the sensitivity to enable high volume manufacturing at current source power output. IM have added a high Z non-metal element onto the crosslinker component of the resist. This also has the benefit of increasing the stiffness of the resist and also enabling a higher post application bake temperature, rising from $80^{\circ} \mathrm{C}$ to $90^{\circ} \mathrm{C}$. The formulation that has been tested also does not include an additional quencher which further reduces the chemical stochastics.

Another method to reduce chemical stochastics by reducing the number of components in the resist formulation is to combine the leaving groups onto the PAG, or alternatively include the epoxy groups, which are currently on a separate crosslinker, on the PAG molecule.

\section{EXPERIMENTAL}

The resist samples were prepared by dissolving the individual components in ethyl lactate or alternatively cyclohexanone. The solutions (using a single solvent) were then combined in various weight ratios and concentrations to give a range of formulations. The resist was spun onto a commercial underlayer, Brewer Scientific E2 STACK AL412302. After spin-coating of the resist the samples received a post application bake (PAB) of $60^{\circ} \mathrm{C}$ for 3 minutes, or $80^{\circ} \mathrm{C}$ or $90^{\circ} \mathrm{C}$ for 1 minute if stated. EUV exposures were performed using the XIL-II interference lithography tool at the Paul Scherrer Institute, Switzerland [7] and using the MET at the Lawrence Berkeley National Laboratory, USA. After exposure the samples did not receive a post exposure bake unless stated, but were developed in n-butyl acetate for 60 seconds followed by 15 seconds of MIBC rinse.

Exposed samples were analyzed with a scanning electron microscope (SEM) in top-down view. Critical dimension (CD) and LER were calculated from the SEM images with the commercial software package SuMMIT. As the direct measurement of the dose at the wafer for PSI exposures was not available in this study, the exposure was measured with respect to an internal PSI reference resist calibrated against exposures of the same resist at the Intel MET [8]. For features 
smaller than the capability of the calibration resist, the dose was estimated from higher pitch sizes. The Berkeley MET dose values were supplied directly as calibrated at the tool.

The baseline for the optimization is the previously introduced xMT resist system, shown in figure 1, from which the MTR2 series resist was developed. The molecular resin has been modified, to increase the glass transition temperature $\left(\mathrm{T}_{\mathrm{g}}\right)$ (MTR2), and additionally increase crosslinkability (MTR3), and adding a higher energy leaving group (MTR4). Alternative crosslinker and photoacid generator molecules were also introduced in the system in order to create more advanced versions of the standard material. Crosslinker CL2 incorporates a non-metal high-Z element for increased optical density. PAG2, a non-antimony PAG, is an option for the standard resist to be used in tracks as a antimony-free material, and also incorporates the standard leaving group onto the PAG.

Triphenylsulfonium tosylate, which acts as a photo-decomposable quencher in epoxy-based systems, was added where indicated. [9]
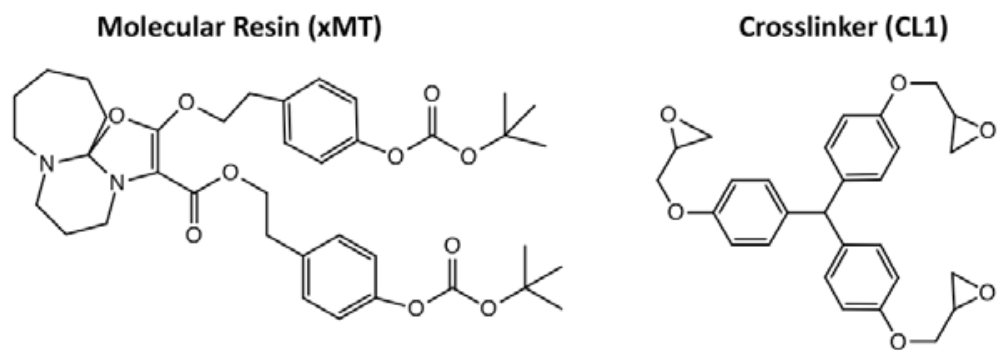

\section{Photo-acid generator (PAG1)}

Figure 3 Resist components in the baseline xMT system

\section{RESULTS}

\subsection{Higher energy leaving groups}

Formulations with the same molar ratios of the base molecule, crosslinker, PAG and quencher were made and patterned at PSI, comparing MTR2 (baseline formulation) and MTR4, which has a higher energy leaving group. Both formulations contained quencher. No PEB was used initially. The results are shown in figures 4 and 5.
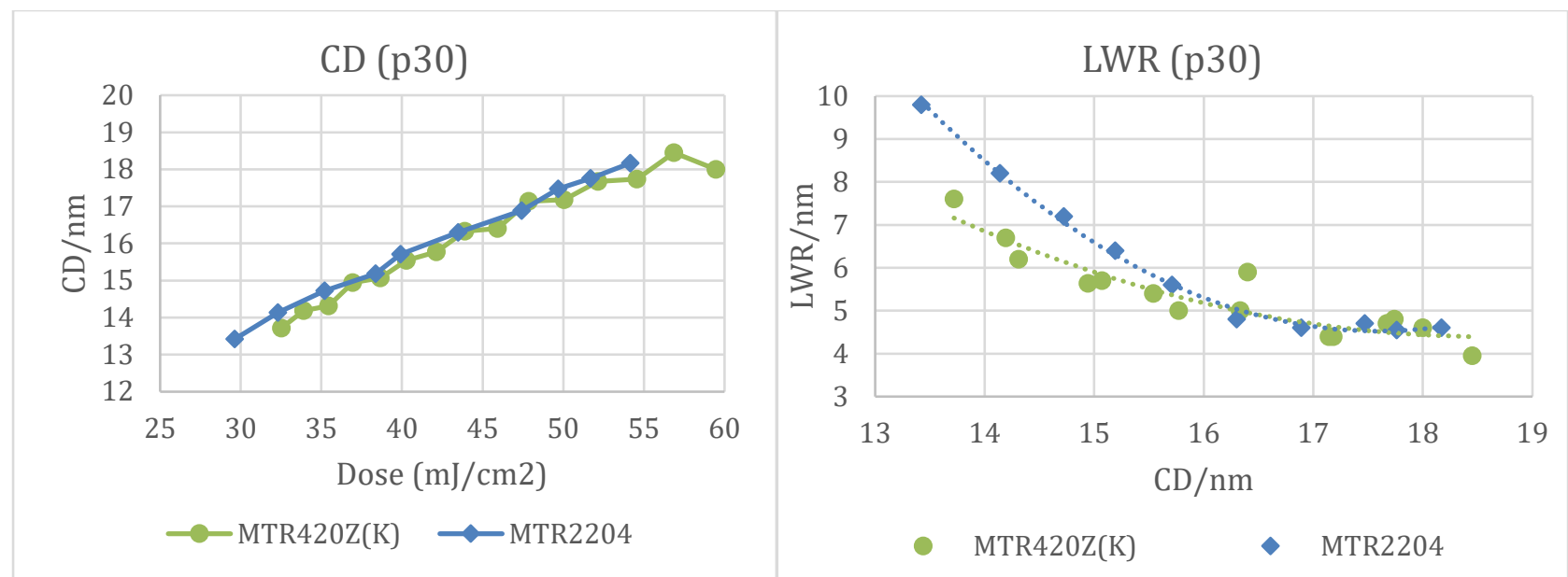

Figure 4: Graphs showing CD and LWR versus dose for MTR2 and MTR4 formulations at pitch 30nm 

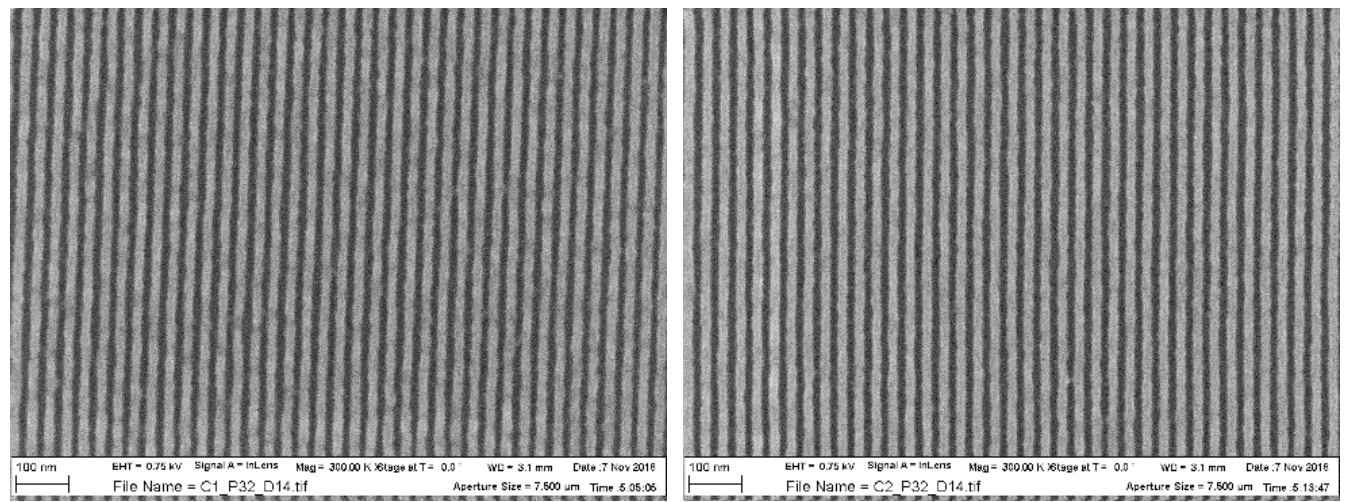

Figure 5: Top-down SEM images of (left) MTR2 at pitch 32nm, 49.3mJ/cm², CD 16.6nm, LER 3.29nm, and (right) MTR4 at pitch 32nm, dose $49.3 \mathrm{~mJ} / \mathrm{cm}^{2}$, CD 16.8nm, LER 2.62nm, patterned at PSI

The dose to size was directly comparable between MTR2 and MTR4. At pitch 30nm, the dose to size for MTR2204 is $37.1 \mathrm{~mJ} / \mathrm{cm}^{2}$ and for MTR420Z(K) is $38.0 \mathrm{~mJ} / \mathrm{cm}^{2}$. At pitch $30 \mathrm{~nm}$, the LWR at the dose to size was $6.6 \mathrm{~nm}$ for MTR2204 and 5.9nm for MTR420Z(K). When a $90^{\circ} \mathrm{C}$ PEB was applied, the dose to size does not change for the standard or higher energy leaving group. The LWR is negatively impacted, the same result as had been reported previously [10], with an average of $23 \%$ increase over all pitches for the standard leaving group, and $19 \%$ for the higher energy leaving group. This indicates that there may be a slight benefit to the higher leaving group but the changes are not yet significant because the difference in energy was not substantial enough. Future study will include adding even higher energy leaving groups.

\subsection{High absorbance non-metallic element}

We have synthesised a crosslinker that contains the high opacity non-metallic atom, CL2, and a new formulation, MTR2620 was created and tested initially at the MET in Berkeley. None of the formulations using CL2 contain quencher. The patterning performance previously presented in [5] and shown in figure 6 (left) shows $17.5 \mathrm{~nm}$ semi-dense lines with low LER values at pitch 48nm. The dose required to pattern these features is higher than desired, therefore further synthesis and formulation work looked at increasing sensitivity.

An alternative formulation ratio using CL2, MTR2627, is designed to improve sensitivity, was patterned at PSI, as shown in figure 6 (right). This shows $12.7 \mathrm{~nm}$ lines patterned at a pitch of $28 \mathrm{~nm}$ with a dose of $53 \mathrm{~mJ} / \mathrm{cm}^{2}$ with an LER of $4.2 \mathrm{~nm}$. The sensitivity of the resist formulation has thus improved, and sub 13nm lines at a dense pitch are possible.
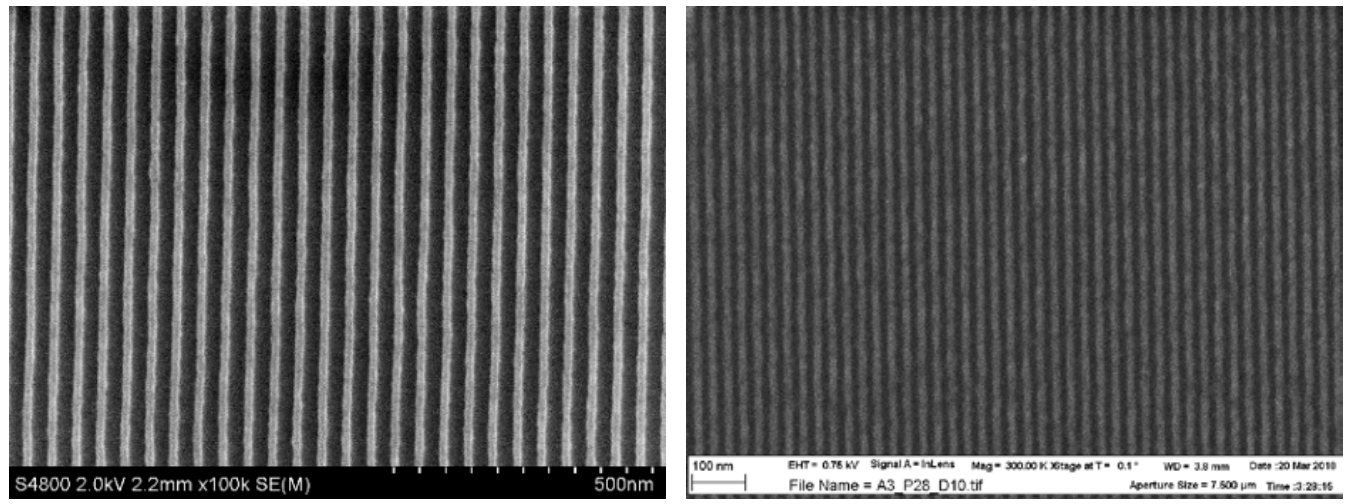

Figure 6: Top-down SEM images of

(left) MTR2620 at pitch 48nm, dose $86.8 \mathrm{~mJ} / \mathrm{cm}^{2}$, CD $17.5 \mathrm{~nm}$, LER $2.80 \mathrm{~nm}$ patterned at Berkeley MET, and (right) MTR2627 at pitch $28 \mathrm{~nm}$, dose $53 \mathrm{~mJ} / \mathrm{cm}^{2}$, CD $12.7 \mathrm{~nm}$, LER 4.2nm, patterned at PSI 
The formulation was changed once more and the MTR ratio increased. MTR262Z(D) was patterned at the MET in Berkeley and the results shown in figure 7 . A PEB of $80^{\circ} \mathrm{C}$ for 1 minute was used.

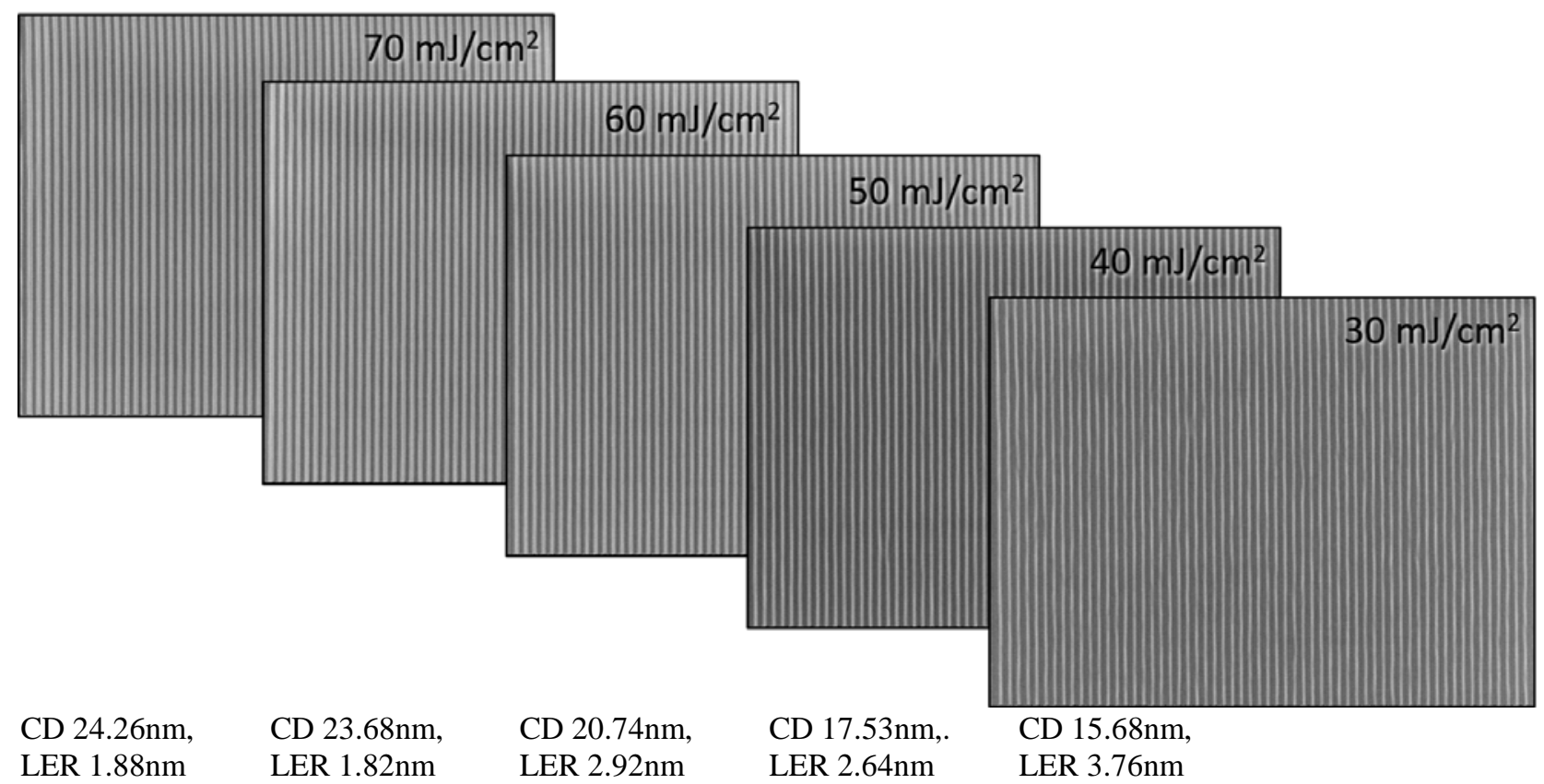

Figure 7: Top down SEMs of MTR262Z(D) at pitch 48nm patterned at Berkeley MET at wide range of doses

An LER of $1.9 \mathrm{~nm}$ were obtained at hp24nm with a dose of $65 \mathrm{~mJ} / \mathrm{cm}^{2}$. For narrow, underexposed features, the LER was still low. Lines with an LER below 4nm can be patterned even when extremely underexposed (i.e. 15.7nm CD at pitch $48 \mathrm{~nm})$. This intriguing feature could potentially be utilized to achieve high resolution at low dose by using a biased mask set. We compared this facet of performance of MTR262Z(D) with the baseline MTR2204 by patterning at PSI and looking at a large range of doses. Figure 8 illustrates the improvement in LWR at exposures giving the correct critical dimension and below when compared to MTR2204.

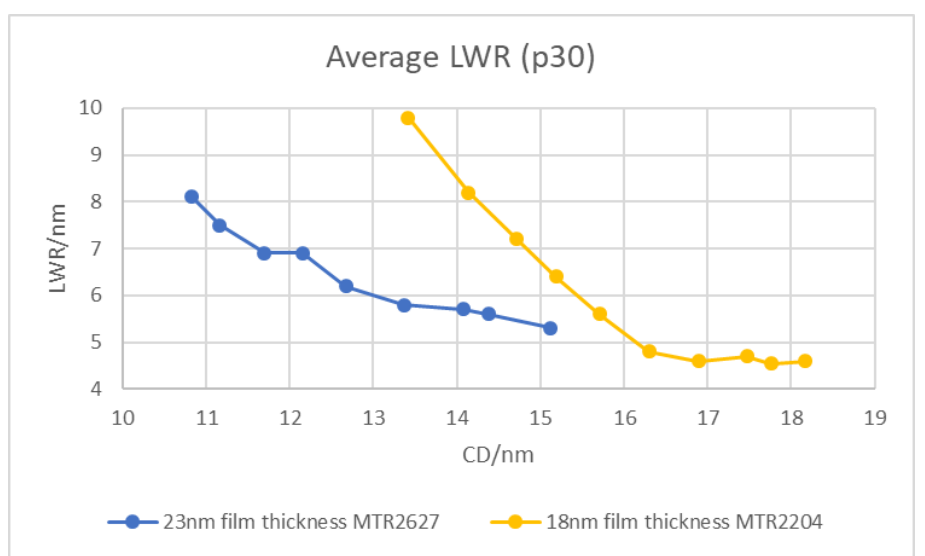

Figure 8: Improvement in LWR at critical dimension and below using high opacity crosslinker compared to standard formulation MTR2204 at pitch 30nm 


\subsection{PEB temperature study with high $\mathrm{Z}$ crosslinker}

MTR2 and MTR4 formulations with the high opacity crosslinker CL2 were studied, using EUV lithography at PSI, in relation to the change in performance with PEB temperature. As shown in figure 9, using a $\mathrm{PEB}\left(90^{\circ} \mathrm{C}\right)$ causes dose-tosize to decrease by $41 \%$ for MTR2. Using a PEB causes LER to increase as we saw previously (section 3.1) using the standard crosslinker.
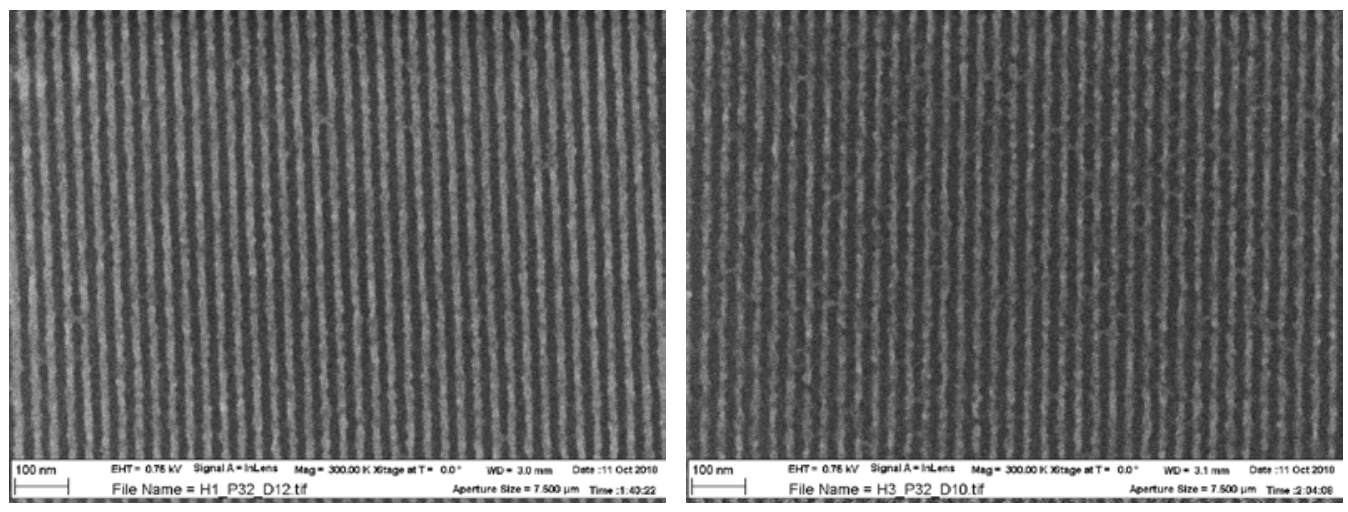

Figure 9; Top down SEM images of dense lines at pitch $32 \mathrm{~nm}$ using MTR262Z(D) formulation, (left) without PEB. dose $65 \mathrm{~mJ} / \mathrm{cm}^{2}$, CD 13.5nm, LER 4.76nm, and (right) with $90^{\circ} \mathrm{C}$ PEB, dose $32 \mathrm{~mJ} / \mathrm{cm}^{2}$, CD 12.6nm, LER 6.70nm

We then investigated this more closely using the MTR462Z(D) formulation, which has the slightly higher energy leaving group and high opacity crosslinker together, by running a set of experiments with a PEB increasing from no PEB up to $90^{\circ} \mathrm{C}$ in $10^{\circ} \mathrm{C}$ intervals, as shown in figure 10 .

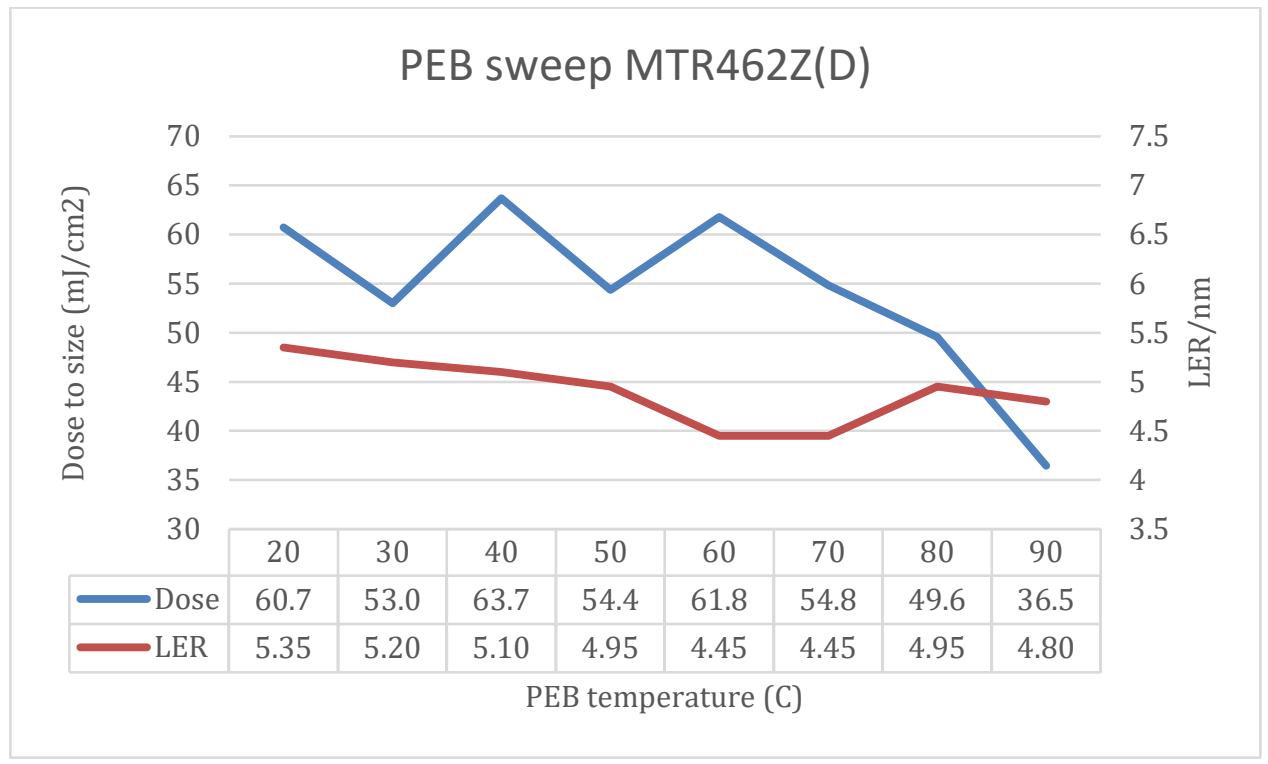

Figure 10: Variation in Dose to size and LER at increasing PEB temperature using formulation MTR462Z(D)

The significant decrease in dose to size starts after $70^{\circ} \mathrm{C}$ although there is some variation at earlier temperatures which we attribute to experimental noise at this stage. The dose required to achieve $16 \mathrm{~nm}$ lines at p32 using a $90^{\circ} \mathrm{C} \mathrm{PEB}$ is $60 \%$ of 
the dose when using no PEB. The LER looks like it is slowly decreasing with increasing temperature, but there is not a sudden change at $80^{\circ} \mathrm{C}$ or $90^{\circ} \mathrm{C}$ in common with the dose change. Thus the higher energy leaving group appears to have a positive impact in this formulation.

\subsection{Use of non-antimony PAG}

In order to eliminate all non-standard elements in our resist, we have synthesized a PAG, PAG2, with no metal or metalloids, based on a weakly-coordinating carbanion, with the MTR leaving groups also incorporated in the cation. The standard PAG used in the resists previously described contains antimony. For the initial tests, the product was not completely pure for these EUV lithography patterning at PSI, but still achieved 16nm lines at p32, as shown in figure 11. The formulation is made without quencher, but still with the MTR2 molecule as well, so was compared against MTR2220, which contains the antimony containing PAG and which also does not contain quencher. The carbon based PAG samples are significantly slower than MTR2220 using the same molar ratio, requiring 2.6x as much dose. It is also slower than the baseline MTR2204, which has quencher, requiring 32\% more dose. The LER is worse than MTR2220 but this is mostly due to the pattern wiggling which we intend to address, initially by purifying the material to the required standard, and by looking at formulation and process variations. Additional weakly coordinating carbanions with even stronger acidity are currently being synthesized.
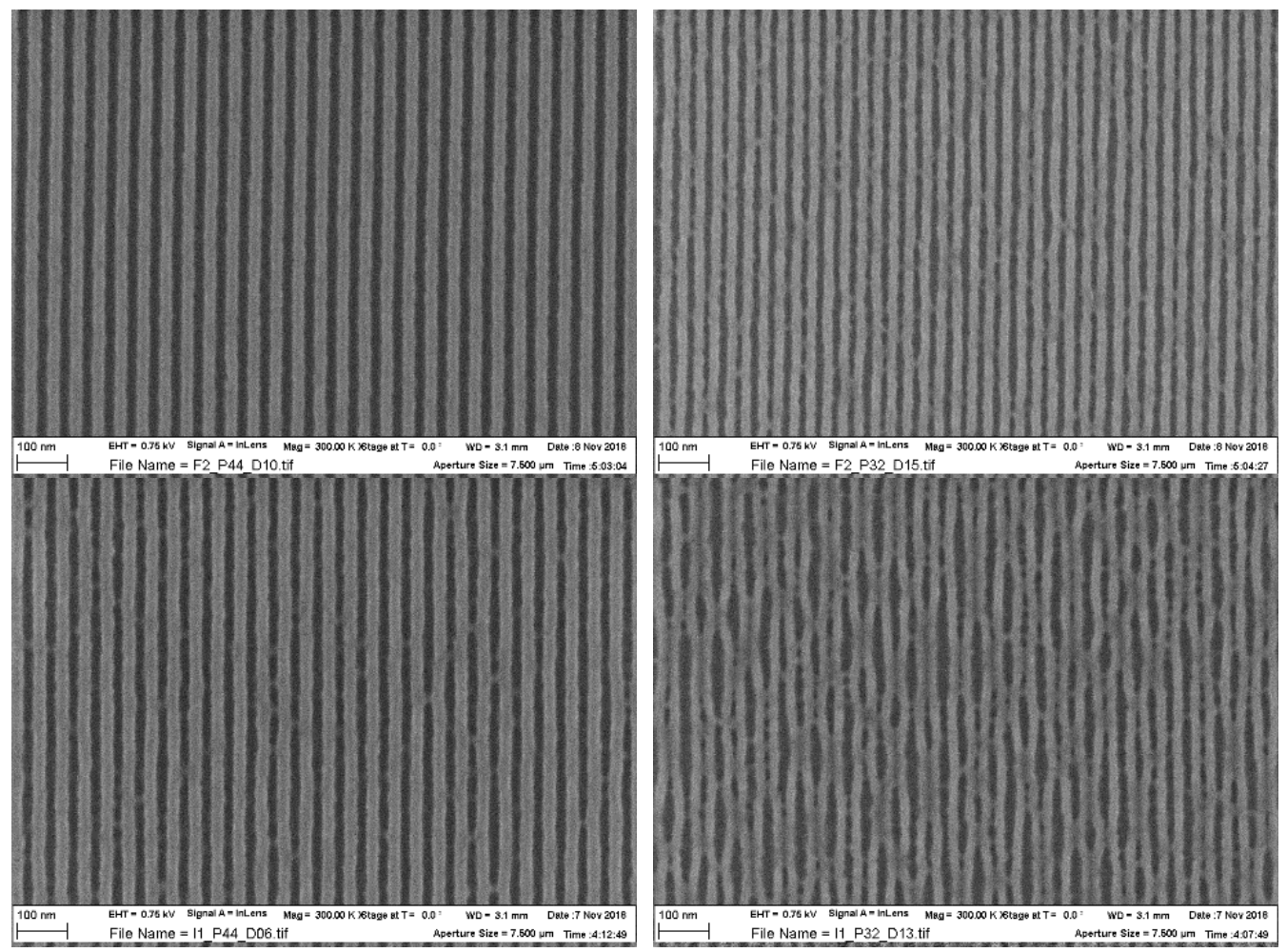

Figure 11: Top down SEM images of dense lines at pitch $44 \mathrm{~nm}$ (left) and pitch 32nm (right) using MTR2220 formulation, (top) (p44) Dose 25mJ/cm², CD 24.3nm, LER 2.51nm; (p32) 25.8mJ/cm², CD 17.9nm, LER 4.14nm; and (bottom) using formulation with carbon based PAG, (p44)Dose 55mJ/cm², CD 25.9nm, LER 3.27nm; and (p32) 61.4mJ/cm², CD 16.8nm, LER $7.70 \mathrm{~nm}$

\section{CONCLUSION}

New synthesis procedures have been developed to produce novel molecules capable of improving the Irresistible Materials Multi Trigger resist which had previously shown 16nm patterning on an NXE3300 scanner. Improved absorption, reduced 
thermal leakage, reduced material stochastics and improved crosslinking have been started to be addressed and the materials' patterning capabilities shown at PSI and BMET using EUV lithography. Additionally, materials which have eliminated metals, specifically the antimony in the PAG molecule, have been developed and tested. A multi trigger resist with a high absorbance non-metal element included in the resist matrix, MTR2627, has been patterned at a pitch of 28nm with an estimated dose of $53 \mathrm{~mJ} / \mathrm{cm}^{2}$ and LER of $4.2 \mathrm{~nm}$. The LWR is improved in the low dose region, and results also show that a thicker film can be used without pattern collapse below pitch 32nm due to increased stiffness. Using the Berkeley MET tool, this resist matrix with a higher MTR ratio has patterned 24nm lines at a pitch of 48nm with an LER of $1.9 \mathrm{~nm}$ with a dose of $65 \mathrm{~mJ} / \mathrm{cm}^{2}$.

\section{ACKNOWLEDGEMENTS}

The authors would like to thank Ms Michaela Vockenhuber from PSI for their assistance with the EUV exposures. Part of this work was performed at Swiss Light Source (SLS), Paul Scherrer Institute, 5232 Villigen, Switzerland. The authors would like to thank the Engineering and Physical Sciences Research Council (EPSRC) for support of this project. The authors thank Irresistible Materials Ltd. and Nano-C for support and provision of resist materials. The Disco DAD 321 wafer dicer used in this research was obtained through the Birmingham Science City provided: Creating and Characterizing Next Generation Advanced Materials, with support from Advantage West Midlands (AWM) and part funded by the European Regional Development Fund (ERDF). C.P. thanks The University of Birmingham for support. This project has received funding from the EU-H2020 research and innovation program under grant agreement No 654360 having benefitted from the access provided by Paul Scherrer Institute in Villigen, Switzerland within the framework of the NFFAEurope Transnational Access Activity.

\section{REFERENCES}

[1] Stowers, J. K., Telecky, A., Kocsis, M., Clark, B. L., Keszler, D. A., Grenville, A., Anderson, C. N., Naulleau, P. P., "Directly patterned inorganic hardmask for EUV lithography,” Proc. SPIE 7969, 796915 (2011).

[2] Krysak, M., Trikeriotis, M., Schwartz, E., Lafferty, N., Xie, P., Smith, B., Zimmerman, P., Montgomery, W., Giannelis, E., Ober, C. K., 'Development of an inorganic nanoparticle photoresist for EUV, e-beam, and $193 \mathrm{~nm}$ lithography,” Proc. SPIE 7972, 79721C (2011).

[3] Cardineau, B., Del Re, R., Al-Mashat, H., Marnell, M., Vockenhuber, M., Ekinci, Y., Sarma, C., Neisser, M., Freedman, D. A., Brainard, R. L., "EUV resists based on tin-oxo clusters," Proc. SPIE 9051, 90511B (2014).

[4] Vesters Y., McClelland A., Popescu C., Dawson G., Roth J., Theis W., de Simone D., Vandenberghe G., Robinson A.P.G, "Multi-trigger resist patterning with ASML NXE3300 EUV scanner," Proc. SPIE 10586, (2018).

[5] Popescu, C., Kazazis, D., McClelland, A., Dawson, G., Roth, J., Theis, W., Ekinci, Y., Robinson, A.P.G., "Highresolution EUV lithography using a multi-trigger resist,” Proc. SPIE 10583, 10583-54 (2018)

[6] Popescu, C., McClelland, A., Kazazis, D., Dawson, G., Roth, R., Ekinci, Y., Theis, W., Robinson, A. P.G., "Multitrigger resist for electron beam and extreme ultraviolet lithography,” Proc. SPIE 10775, 10775-02 (2018).

[7] Päivänranta B., Langner A., Kirk E., David C., Ekinci Y., "Sub-10 nm patterning using EUV interference lithography," Nanotechnology 22, 375302 (2011)

[8] Ekinci Y., Vockenhuber M., Terhalle B., Hojeij M., Wang L., Younkin T. R., "Evaluation of resist performance with EUV interference lithography for sub-22 nm patterning,” Proc. SPIE 8322, 83220W (2012).

[9] Lawson R.A., Frommhold A., Yang D.X., Robinson A.P.G, "Negative-tone organic molecular resists," in Robinson A.P.G., Lawson R.A. editors. Materials and Processes for Next Generation Lithography. Oxford, Elsevier (2016).

[10] Montgomery, W., McClelland A., Ure D., Roth J., Robinson A., "Irresistible Materials multi-trigger resist: the journey towards high volume readiness,” SPIE 10143, 1014328 (2017). 